\title{
Reparación de hernias paraesofágicas gigantes asistida por robot en un hospital de cuarto nivel en Bogotá, Colombia, Clínica Shaio: serie de casos y revisión de la literatura
}

\author{
Robot assisted giant paraesophageal hernias repair in a fourth level hospital in Bogotá, \\ Colombia, Shaio Clinic: case series and literature review
}

\author{
Rubén Luna, ${ }^{1,2}$, Carlos Luna-Jaspe ${ }^{2,3}$, Angie K. Pérez-Hoyos ${ }^{2,4 *}$, Luis F. Cabrera ${ }^{2,5}$, Mauricio Pedraza ${ }^{2,4}$, \\ Jean A. Pudilo ${ }^{2,4}$, Laura T. Padilla, ${ }^{2,6}$, Marcia Santafée, ${ }^{2,4}$ Steven Aparicio 2,4 \\ ${ }^{1}$ Departamento de Cirugía General, de Trasplantes y Robótica, Clínica Shaio; ${ }^{2}$ Escuela de Medicina, Universidad El Bosque; ${ }^{3}$ Departamento de \\ Cirugía General y Bariátrica, Clínica Shaio; ${ }^{4}$ Departamento de Cirugía General, Universidad El Bosque; ${ }^{5}$ Departamento de Cirugía General, Los \\ Cobos Medical Center; ${ }^{6}$ Departamento de Cirugía Pediátrica, Hospital de la Misericordia. Bogotá, Colombia
}

\begin{abstract}
Resumen
Antecedentes: Las hernias paraesofágicas gigantes tienen indicación quirúrgica en caso de ser sintomáticas. Hace 20 años se incorporó la reparación asistida por robot debido a las ventajas que ofrece sobre las limitantes de la laparoscopia. Objetivo: Reportar la experiencia en reparación de hernias paraesofágicas gigantes asistida por robot en un hospital de cuarto nivel en Bogotá, Colombia, Fundación Clínica Shaio. Método: Estudio retrospectivo, descriptivo, de cinco casos de hernia paraesofágica gigante tipo III o IV, Ilevados a corrección robótica, de agosto de 2016 a junio de 2018, con evaluación de los desenlaces posoperatorios. Resultados: Cinco pacientes fueron sometidos a reparación asistida por robot, el tiempo quirúrgico fue de 146 minutos, se realizó una sola conversión a cirugía abierta, la media de sangrado fue de $100 \mathrm{ml}$, el tiempo de estancia hospitalaria de 2.2 días, y los porcentajes de morbilidad, mortalidad y recurrencia a 1 año fueron del $0 \%$. Conclusión: La reparación de este tipo de hernias asistida por robot ha mostrado ventajas sobre el abordaje laparoscópico, como realizar disecciones en ángulos de difícil acceso, precisión aumentada, ergonomía y vista tridimensional y más cercana del espacio de trabajo. Además, promueve mejores desenlaces quirúrgicos y posoperatorios, ventajas evidenciadas principalmente en la reparación de hernias paraesofágicas gigantes.
\end{abstract}

Palabras clave: Cirugía laparoscópica. Cirugía robótica. Hernia paraesofágica. Hernia paraesofágica gigante.

\begin{abstract}
Background: Giant paraesophageal hernias have a surgical indication in case of symptoms. Since twenty years ago robotassisted repair was incorporated to overcome the limitations of the laparoscopic surgery, and to offer new advantages. Objective: To report the experience on repairing giant paraesophageal hernias assisted by robot in a fourth level hospital in Bogotá, Colombia, Shaio Clinic. Method: Retrospective and descriptive study of five cases of giant paraesophageal hernia type III or IV, taken to robotic correction during August 2016 to June 2018. Evaluation of post-surgery outcomes. Results: Five paraesophageal robot-assisted repair were performed. Mean surgical time was 146 minutes, one conversion to open surgery,
\end{abstract}

\footnotetext{
Correspondencia:

*Angie K. Pérez-Hoyos

Avda. Cra 9 No. 131 A-02

Usaquén

Fecha de recepción: 19-10-2019

Cir Cir. 2020;88(6):732-737

C.P 110121, Bogotá, Colombia

Fecha de aceptación: 20-08-2020

Contents available at PubMed

E-mail: akperez@unbosque.edu.co

DOI: 10.24875/CIRU.19001664

www.cirugiaycirujanos.com

0009-7411/C 2020 Academia Mexicana de Cirugía. Publicado por Permanyer. Este es un artículo open access bajo la licencia CC BY-NC-ND (http://creativecommons.org/licenses/by-nc-nd/4.0/).
} 
the average intraoperative bleeding was $100 \mathrm{~mL}$, hospital stay time of 2.2 days. Morbidity, mortality and recurrence percentages in the short time were equal to $0 \%$. Conclusions: Robot-assisted repair of giant paraesophageal hernias, has shown advantages that overcome the limitations of the laparoscopic approach such as dissections in difficult-to-reach angles, increased accuracy, ergonomics, three-dimensional, and closer view of the workspace. In addition, robot-assisted repair promotes better surgical and postoperative outcomes; these advantages have been demonstrated mainly in the repair of giant paraesophageal hernias.

Key words: Laparoscopic surgery. Robotic surgery. Paraesophageal hernia. Giant paraesophageal hernia.

\section{Introducción}

Las hernias paraesofágicas corresponden al 5-10\% de todas la hernias de hiato ${ }^{1-4}$. No existe una definición establecida sobre las hernias paraesofágicas gigantes, siendo consideradas como la herniación de más del $30-50 \%$ del estómago o bien aquellas con un anillo herniario de $5 \mathrm{~cm}$ o más ${ }^{1}$. Para este tipo de hernias, el tratamiento conservador (observación) es una alternativa en los pacientes asintomáticos ${ }^{1,4}$, mientras que para los sintomáticos está indicada la reparación quirúrgica, debido a que se asocian con complicaciones potencialmente mortales (torsión, perforación, hemorragia y gangrena) $)^{1,2,5}$.

La laparoscopia es el abordaje más utilizado 2,3,5-8 por sus menores tasa de complicaciones relacionadas con la incisión, tiempo quirúrgico, estancia hospitalaria y tiempo de recuperación ${ }^{1-3,8}$. Sin embargo, presenta limitaciones, como reducción de la destreza y control visual deficiente, que deben ser tenidas en cuenta al reparar este tipo de hernias ${ }^{9}$. A partir de los años 1990 se introdujeron sistemas robóticos que han demostrado superioridad frente a estas limitantes de la laparoscopia ${ }^{9}$, aportando imágenes tridimensionales ${ }^{2-5,7-11}$, de alta definición, brazos operativos estables $^{2,3,7-9}$ y mejores condiciones ergonómicas ${ }^{3,5,8,9,12}$, lo cual minimiza las complicaciones intraoperatorias ${ }^{7}$.

Algunos estudios con bajo peso estadístico indican que el abordaje robótico no es superior al laparoscópico $2,7,8,13$. Sin embargo, se ha evidenciado que se asocia con una tasa más baja de recurrencia ${ }^{2,4,5}$ y menores complicaciones ${ }^{2,3}$. Dichos estudios se han realizado con un número pequeño de pacientes y con un seguimiento relativamente corto $^{2}$, por lo que se requieren ensayos más grandes que comparen ambas vías de abordaje para establecer recomendaciones con respecto a eficacia, resultados y rentabilidad $2,3,7,8,10$. Nos permitimos mostrar nuestra experiencia en el manejo robótico de las hernias paraesofágicas gigantes en un hospital de cuarto nivel en Bogotá, Colombia, y realizar una revisión crítica de la literatura.

\section{Método}

Se llevó a cabo un estudio retrospectivo, descriptivo, incluyendo todos los pacientes con hernia paraesofágica gigante tipo III o IV llevados a corrección robótica de forma programada o de urgencia, durante agosto de 2016 a junio de 2018, en un hospital de cuarto nivel en Bogotá, Colombia, Clínica Shaio. Se evaluaron la edad, el sexo, el sangrado, las infecciones, las complicaciones, la conversión a laparoscopia, la conversión a cirugía abierta, el tiempo quirúrgico, la estancia en la unidad de cuidados intensivos, la estancia hospitalaria y la mortalidad.

El protocolo de estudio fue aprobado por el comité de ética de las instituciones. El protocolo fue implementado de conformidad con las disposiciones de la Declaración de Helsinki y las guías de buena práctica clínica, garantizando así el mismo esquema de manejo independientemente de la institución y del paciente.

Por ser un estudio observacional retrospectivo de cohorte, se realizó un análisis de los datos usando SPSS1 (Statistical Package For The Social Sciences) versión 22.0, y se calcularon rangos, medianas, mínimas, máximas y porcentajes.

Los pacientes fueron colocados en decúbito supino con ambos brazos cerrados. El procedimiento se realizó utilizando cinco puertos. Se colocó un trocar Optiview de $12 \mathrm{~mm}$ con un laparoscopio de $0^{\circ}$ en posición supraumbilical para obtener acceso a la cavidad peritoneal bajo visualización directa, y se creó el neumoperitoneo con dióxido de carbono a una presión de 12-15 mmHg. Se colocó un puerto de $5 \mathrm{~mm}$ en el epigastrio y, a través de él, se introdujo un separador de hígado para retraer el lóbulo izquierdo hepático y exponer el hiato esofágico. Se colocó un segundo puerto de $12 \mathrm{~mm}$ (utilizado como puerto auxiliar) en el área subcostal izquierda. Se colocaron otros dos puertos de $8 \mathrm{~mm}$, uno a la derecha del ombligo y el otro en la línea medioclavicular izquierda, los cuales 
funcionaron como puertos de trabajo robóticos. Después de esto, el robot quedó atracado por encima del hombro izquierdo del paciente. Todos los instrumentos se insertaron bajo visión directa para evitar lesiones involuntarias en las vísceras abdominales. Luego, el cirujano comenzó la disección desde la consola robótica. El contenido de la hernia se redujo para exponer el hiato. Se retiró el ligamento gastrohepático, preservando la vasculatura hepática aberrante, y se expuso la crura derecha. La disección del saco se inició en el pilar derecho y este se separó del tejido mediastinal con disección atraumática, mientras se dividió circunferencialmente en el orificio hiatal. Los vasos gástricos cortos fueron disecados y seccionados para exponer el pilar izquierdo y completar la disección circunferencial del saco, con el fin de reducirlo completamente hacia el abdomen, sin dejar ninguna porción ni contenido del saco dentro del tórax. Posteriormente, se creó una ventana retroesofágica y se avanzó un drenaje Penrose, rodeando por completo el esófago, con el fin de utilizarlo para realizar una tracción esofágica atraumática. Se finalizó la disección esofágica hasta movilizar el esófago completamente y lograr una buena longitud intraabdominal. De alrededor de $3 \mathrm{~cm}$. Se procedió a realizar una reparación posterior de las cruras, con puntos separados antitensión, utilizando una sutura sintética de poliéster multifilamento trenzada del n. 2 (Ethibond $^{\circledR}$, Ethicon, Inc., Cincinnati, USA), con anudado intracorpóreo, calibrando el hiato con una bujía de 60 Fr. Se colocó una malla de poliéster recubierta de colágeno (Parietex ${ }^{\top M}$ Composite Hiatal, Medtronic Inc., Minneapolis, USA) tipo onlay, reforzando el defecto previamente reparado, rodeando el esófago intraabdominal, la cual se fijó al diafragma con puntos simples cardinales utilizando una sutura sintética de poliéster multifilamento trenzada del n. ${ }^{0} 2$ (Ethibond $^{\circledR}$, Ethicon, Inc., Cincinnati, USA), con anudado intracorpóreo. En caso de ser insuficiente la longitud esofágica, se adicionó una gastroplastia tipo Collis. Por último, se llevó a cabo una funduplicatura tipo floppy Nissen de $360^{\circ}$ sobre la bujía esofágica de $60 \mathrm{Fr}$, con tres puntos simples separados de sutura sintética de poliéster multifilamento trenzada del n. ${ }^{0} 2$ (Ethibond $^{\circledR}$, Ethicon, Inc., Cincinnati, USA), con anudado intracorpóreo del fundus gástrico-esófago-fundus gástrico. En todos los pacientes que presentaron vólvulo gástrico se colocó un tubo de gastrostomía para gastropexia. Se realizó y revisó la hemostasia. Al finalizar, el robot se desacopló después de quitar el retractor del hígado. Se extrajeron los puertos bajo visión
Tabla 1. Resultados de la intervención quirúrgica

\begin{tabular}{lc}
\hline Tiempo quirúrgico & $146 \mathrm{~min}$ \\
Estancia hospitalaria & 2.2 días \\
Tasa de conversión, $\mathrm{n}(\%)$ & $1(20)$ \\
Complicaciones, $\mathrm{n}(\%)$ & $0(0)$ \\
Recurrencia, $\mathrm{n}(\%)$ & $0(0)$ \\
Mortalidad, $\mathrm{n}(\%)$ & $0(0)$ \\
\hline
\end{tabular}

directa, se retiró el neumoperitoneo y se cerró la aponeurosis con puntos simples separados de polidoxanona del n. 0 (PDS $\AA$, Ethicon, Inc., Cincinnati, USA) en los puertos de 8 y $12 \mathrm{~mm}$. Se cerró la piel con puntos intradérmicos de polipropileno del n. ${ }^{\circ} 3$ (Prolene ${ }^{\circledR}$, Ethicon, Inc., Cincinnati, USA). A las 24 horas de posoperatorio se realizó un estudio de vías digestivas altas con contraste hidrosoluble y se inició la vía oral con dieta líquida. Se dio el alta hospitalaria al segundo día y se realizó un nuevo estudio de vías digestivas altas con contraste hidrosoluble al mes, con seguimiento clínico de forma ambulatoria.

\section{Resultados}

Entre agosto de 2016 y junio de 2018, cinco pacientes fueron sometidos a reparación de hernia paraesofágica gigante (tipo IV) asistida por robot. La edad media de los pacientes fue de 57 años y el $60 \%$ eran mujeres. Todos los pacientes presentaban síntomas secundarios a esta patología. En cuatro de ellos se llevó a cabo la cirugía de forma electiva y en uno fue realizada de urgencias. El tiempo quirúrgico promedio fue de 146 minutos, incluyendo el tiempo de acoplamiento del robot (Tabla 1). Se realizó una conversión a cirugía abierta debido a dificultades anatómicas; no hubo conversión a cirugía laparoscópica. El sangrado promedio fue de $100 \mathrm{ml}$. No se presentaron complicaciones posoperatorias inmediatas ni en los 30 días de seguimiento. Los porcentajes de morbilidad, mortalidad y recurrencia a 1 año fueron del $0 \%$, y la estancia hospitalaria promedio fue de 2.2 días.

\section{Discusión}

Las hernias hiatales son afecciones de resolución quirúrgica relativamente frecuentes y se clasifican en cuatro grupos, dependiendo de la localización de la 
Tabla 2. Clasificación de las hernias hiatales ${ }^{13}$

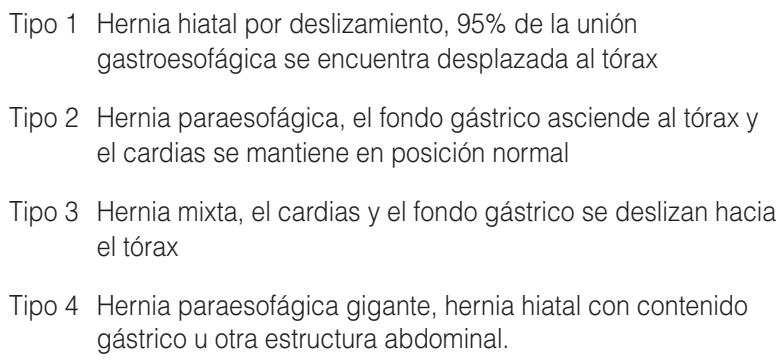

Tipo 1 Hernia hiatal por deslizamiento, 95\% de la unión gastroesofágica se encuentra desplazada al tórax

Tipo 2 Hernia paraesofágica, el fondo gástrico asciende al tórax y el cardias se mantiene en posición normal

Tipo 3 Hernia mixta, el cardias y el fondo gástrico se deslizan hacia el tórax

Tipo 4 Hernia paraesofágica gigante, hernia hiatal con contenido gástrico u otra estructura abdominal.

unión gastroesofágica, el contenido y la presencia de saco herniario, como se muestra en la tabla $2^{2,5,8,11}$. Las hernias hiatales gigantes son hernias mixtas (paraesofágicas y deslizadas) en las que más del 30-50\% del estómago tiene una ubicación intratorácica'.

Las hernias paraesofágicas gigantes pueden ser primarias o secundarias a un procedimiento quirúrgico, como cirugía antirreflujo, corrección de hernias hiatales o esofagectomía. La laparoscopia es uno de los factores de riesgo identificados para su presentación'. Se ven favorecidas por la presión torácica negativa y la presión positiva en el abdomen'.

La presentación clínica puede ir desde un cuadro asintomático hasta una presentación aguda o cróni$\mathrm{ca}^{1}$. El $0.7-7 \%$ de los pacientes experimentan síntomas de un cuadro agudo (dolor torácico, vómito y disnea), los cuales se deben a complicaciones como vólvulo gástrico, hemorragia, estrangulación o perforación ${ }^{1}$. Los síntomas del cuadro crónico se encuentran relacionados con la obstrucción gástrica 0 esofágica, o con la presencia de reflujo gastroesofágico'. Dentro de estos síntomas se encuentran disfagia, disnea, náuseas, vómitos y anemia'. Todos los pacientes presentados se encontraban sintomáticos, por lo cual fueron llevados a reparación quirúrgica.

Los estudios del tracto gastrointestinal alto brindan información sobre el tamaño y el tipo de hernia, la localización de la unión esofagogástrica, la presencia de obstrucción esofágica y la longitud del esófago'. Dentro de estos estudios se encuentran la endoscopia y la manometría esofágica (realizada de forma completa en menos del $50 \%$ de los pacientes) ${ }^{1}$. En los cuadros agudos, mediante tomografía computarizada y radiografía de tórax se pueden descartar perforación, neumomediastino y neumoperitoneo, y se pueden observar el tipo y la localización de la hernia y la perfusión de la pared gástrica'.

Dentro del manejo inicial del paciente estable se encuentra la descompresión gástrica, realizada con
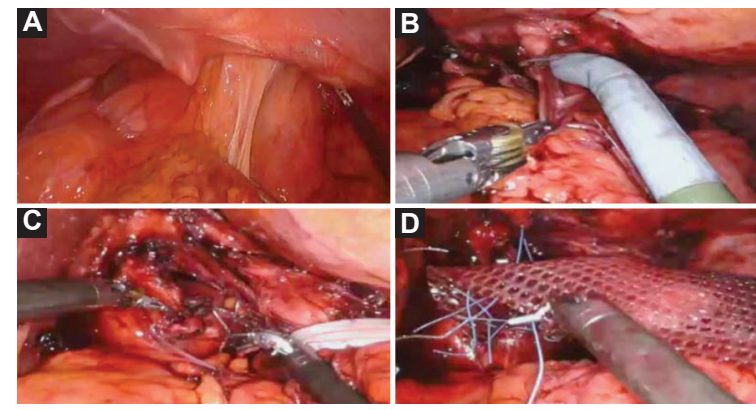

Figura 1. Reparación de hernia paraesofágica asistida por robot. A: exposición del hiato esofágico. B: disección de los pilares del diafragma. C: cierre del hiato esofágico. D: refuerzo de la reparación de los pilares con malla.

una sonda nasogástrica bajo guía endoscópica, para evaluar la permeabilidad de la unión esofagogástrica y la ausencia de isquemia'. En caso de inestabilidad o de signos de isquemia o perforación gástrica, la cirugía no se puede retrasar y hay que intentar el abordaje laparoscópico como primera opción ${ }^{1}$, en la cual se deben realizar la reducción de la hernia, la resección del saco herniario, la movilización esofágica, el reposicionamiento de la unión esofagogástrica, la reparación crural y el procedimiento antirreflujo ${ }^{6}$. En nuestros pacientes, el procedimiento que se llevó a cabo inició con la exposición del hiato y siguió con la reducción de la hernia, la disección de los pilares del diafragma, el cierre del hiato esofágico y el refuerzo de la reparación de los pilares con malla (Fig. 1).

La cirugía robótica cada vez es más usada en la cirugía general, lo que se puede evidenciar en publicaciones que hablan acerca de la seguridad y la viabilidad de este tipo de abordaje en procedimientos como la colecistectomía, la gastrectomía, la cirugía colorrectal y la cirugía pancreática ${ }^{3}$. Los reportes en la literatura sobre la reparación de hernias paraesofágicas por robot datan de hace aproximadamente 20 años ${ }^{1}$.

Algunos estudios ${ }^{1}$ han realizado comparaciones entre la vía de abordaje abierta y la laparoscópica, y han evidenciado que la reparación por laparoscopia se relaciona con una menor estancia hospitalaria (3.74.2 vs. 8.3-8.5 días), una disminución significativa de la tasa de lesiones intraoperatorias y de la mortalidad hospitalaria (0.6 vs. $3.0 \%$ ), un menor requerimiento de ingreso en la unidad de cuidados intensivos (13 vs. $35 \%$ ), un menor porcentaje de complicaciones ( 2.7 vs. $8.4 \%$ ), menos readmisiones a 30 días (1.4 vs. $3.4 \%$ ) y una reducción de los costos $(\$ 15,227$ vs. $\$ 24,263)$; con $p<0.01$. 
Con respecto a la reparación asistida por robot, Ruurda, et al. ${ }^{9}$ señalan que la disección del saco herniario y la reparación crural son más fáciles según su experiencia de 4 años de reparación asistida por robot. Estos autores indican que, en promedio, el tiempo quirúrgico fue de 130 minutos (rango: 82-210) y la estancia hospitalaria fue de 5 días (rango: 2-39). Presentaron tres casos de conversión, una tasa de complicaciones del 0-28\% y una tasa de reoperación del $12.5 \%$. Además, tuvieron tres casos de reoperación en el posoperatorio temprano, debidos a drenaje abierto de absceso, reparo de perforación esofágica y cruroplastia fallida con nueva herniación del estómago, respectivamente. Al comprarlo con nuestra experiencia, nosotros presentamos un tiempo quirúrgico similar y menores estancia hospitalaria, tasa de conversión y tasa de recurrencia.

Gehrig, et al. ${ }^{8}$ compararon las reparaciones abierta, laparoscópica y asistida por robot. No encontraron diferencia significativa con respecto al tiempo quirúrgico. La tasa de complicaciones intraoperatorias y posoperatorias fue menor con el abordaje asistido por robot $(16.7 \%)$ y en el laparoscópico $(17.6 \%)$ con respecto al abierto $(58.3 \%)^{8}$. En este estudio, la estancia hospitalaria fue significativamente menor para los procedimientos mínimamente invasivos.

Por medio de la corrección asistida por robot se ha observado un tiempo quirúrgico de 125-212 minutos (rango: 80-258), un sangrado de 25-50 ml (rango: 0-700), una estancia hospitalaria de 1.4-4.5 días (rango: 1-39), un $5 \%$ de complicaciones intraoperatorias (laceración hepática y perforación esofágica), un $12.5 \%$ de complicaciones posoperatorias (relacionadas con la curva de aprendizaje) y un $7.5 \%$ de reoperaciones en 30 días (hernia estrangulada, perforación esofágica, recurrencia de la hernia hiatal debido a dehiscencia de la hiatoplastia). El $15.6 \%$ de los pacientes presentaron síntomas de disfagia tras la operación y el 91\% estaban satisfechos con los resultados de la cirugía al año,10,12. Se puede observar que, en nuestros casos, el tiempo quirúrgico se encuentra dentro del rango, al igual que la estancia hospitalaria, pero la tasa de complicaciones es menor.

Tolboom, et al. ${ }^{11}$ realizaron un estudio observacional en el que compararon la corrección redo de hernias hiatales por vía laparoscópica o asistida por robot. En el grupo asistido por robot hubo una menor conversión a laparotomía (1/45 vs. $5 / 30 ; p=0.035)$ y una menor estancia hospitalaria (3 [2-6] vs. 4 [3-7]; $p=0.042$ ). No hubo una diferencia significativa con respecto a la estancia hospitalaria ni a los síntomas posoperatorios entre los dos grupos. Estos autores indican que la cirugía redo es más exigente, debido a la presencia de adherencias o de cambios anatómicos o de planos, por lo que se benefician del abordaje asistido por robot.

\section{Conclusiones}

La vía de abordaje recomendada hasta el momento para la reparación de hernias paraesofágicas es la laparoscópica, pero se ha evidenciado que mediante la reparación asistida por robot existen ventajas que promueven mejores desenlaces quirúrgicos y posoperatorios en casos de hernias gigantes, con estómago al revés, recurrencia o incluso en situación de emergencia (encarcelamiento o estrangulación), gracias a la capacidad de realizar disecciones en ángulos de difícil acceso, su precisión aumentada, la mejor ergonomía y proporcionar una vista tridimensional y más cercana del espacio de trabajo. Sin embargo, se requieren estudios comparativos con amplias muestras y un seguimiento a largo plazo que permitan confirmar estos hallazgos.

\section{Agradecimientos}

Los autores agradecen al Dr. Rubén Luna y a la Fundación Clínica Shaio por permitir publicar los casos presentados.

\section{Financiamiento}

Los autores no tuvieron ningún financiamiento.

\section{Conflicto de intereses}

Los autores declaran no tener ningún conflicto de intereses que declarar.

\section{Responsabilidades éticas}

Protección de personas y animales. Los autores declaran que para esta investigación no se han realizado experimentos en seres humanos ni en animales.

Confidencialidad de los datos. Los autores declaran que han seguido los protocolos de su centro de trabajo sobre la publicación de datos de pacientes.

Derecho a la privacidad y consentimiento informado. Los autores han obtenido el consentimiento informado de los pacientes y/o sujetos referidos en el artículo. Este documento obra en poder del autor de correspondencia. 


\section{Bibliografía}

1. Dallemagne B, Quero G, Lapergola A, Guerriero L, Fiorillo C, Perretta S. Treatment of giant paraesophageal hernia: pro laparoscopic approach. Hernia. 2018;22:909-19.

2. Seetharamaiah R, Romero RJ, Kosanovic R, Gallas M, Verdeja JC Rabaza J, et al. Robotic repair of giant paraesophageal hernias. JSLS. 2013;17:570-7.

3. Vasudevan V, Reusche R, Nelson E, Kaza S. Robotic paraesophagea hernia repair: a single-center experience and systematic review. J Robotic Surgery. 2018;12:81-6.

4. Draaisma WA, Gooszen HG, Consten EC, Broeders IA. Mid-term results of robot-assisted laparoscopic repair of large hiatal hernia: a symptomatic and radiological prospective cohort study. Surg Technol Int. 2008:17:165-70

5. Morelli L, Guadagni S, Mariniello MD, Pisano R, D'Isidoro C Belluomini MA, et al. Robotic giant hiatal hernia repair: 3 year prospective evaluation and review of the literature. Int J Med Robot. 2015;11:1-7.

6. Brenkman HJ, Parry K, van Hillegersberg R, Ruurda JP. Robot-assisted laparoscopic hiatal hernia repair: promising anatomical and functional results. J Laparoendosc Adv Surg Tech A. 2016;26:465-9.
7. Galvani CA, Loelb H, Osuchukwu O, Samamé J, Apel ME, Ghaderi I. Robotic-assisted paraesophageal hernia repair: initial experience at a single institution. J Laparoendosc Adv Surg Tech A. 2016;26:290-5.

8. Gehrig T, Mehrabi A, Fischer L, Kenngott H, Hinz U, Gutt CN, et al. Robotic-assisted paraesophageal hernia repair - a case-control study. Langenbecks Arch Surg. 2013;398:691-6.

9. Ruurda JP, van Vroonhoven TJ, Broeders IA. Robot-assisted surgical systems: a new era in laparoscopic surgery. Ann R Coll Surg Engl. 2002; 84:223-6.

10. Dunnican WJ, Singh TP, Guptill GG, Doorly MG, Ata A. Early robotic experience with paraesophageal hernia repair and Nissen fundoplication: short-term outcomes. J Robotic Surg. 2008;2:41-4.

11. Tolboom RC, Draaisma WA, Broeders IA. Evaluation of conventional laparoscopic versus robot-assisted laparoscopic redo hiatal hernia and antireflux surgery: a cohort study. J Robot Surg. 2016;10:33-9.

12. Sarkaria IS, Latif MJ, Bianco VJ, Bains MS, Rusch VW, Jones DR, et al. Early operative outcomes and learning curve of robotic assisted giant paraesophageal hernia repair. Int J Med Robot. 2017;13:10.1002/rcs.1730.

13. Villareal R, Bernal F, Cabrera LF, Sánchez Ussa S, Pedraza M. Hernia hiatal mixta con vólvulo gástrico intratorácico: reporte de caso y revisión de la literatura. Rev Hispanoam Hernia. 2018;6:186-90. 